\title{
Twice $Q$-polynomial distance-regular graphs of diameter 4
}

\author{
Jianmin $\mathrm{Ma}^{\mathrm{a}}$, Jack H. Koolen ${ }^{\mathrm{b}, *}$ \\ ${ }^{a}$ Hebei Key Lab of Computational Mathematics $\&$ Applications and College of Math 8 Info. Science \\ Hebei Normal University, Shijiazhuang, Hebei 050016, China \\ ${ }^{b}$ School of Mathematical Sciences, University of Science and Technology of China, Hefei, Anhui 230026, China
}

\begin{abstract}
It is known that a distance-regular graph with valency $k$ at least three admits at most two Q-polynomial structures. In this note we show that all distance-regular graphs with diameter four and valency at least three admitting two $Q$-polynomial structures are either dual bipartite or almost dual imprimitive. By the work of Dickie [5] this implies that any distance-regular graph with diameter $d$ at least four and valency at least three admitting two $Q$-polynomial structures is, provided it is not a Hadamard graph, either the cube $H(d, 2)$ with $d$ even, the half cube $1 / 2 H(2 d+1,2)$, the folded cube $\tilde{H}(2 d+1,2)$, or the dual polar graph on $\left[{ }^{2} A_{2 d-1}(q)\right]$ with $q \geq 2$ a prime power.
\end{abstract}

Keywords: Distance-regular graph, $P$ - or $Q$-polynomial structure, tight

2008 MSC: 05E30

\section{Introduction}

Dickie [5] showed that any distance-regular graph with diameter at least four and valency at least three admits at most two $Q$-polynomial structures, extending the work of Bannai and Ito who showed it for diameter at least 34. For brevity we call a distance-regular graph (or an association scheme) with exactly two $Q$-polynomial structures twice Q-polynomial. Furthermore, Dickie [5] classified twice $Q$-polynomial distance-regular graphs with diameter at least five and valency at least three.

Theorem 1. [5, Theorem 8.1.2] Let $\Gamma$ be a distance regular graph with diameter $d \geq 5$ and valency $k \geq 3$. Then $\Gamma$ has two Q-polynomial structures if and only if $\Gamma$ is one of the following:

(i) the cube $H(d, 2)$ with $d$ even;

(ii) the half cube $\frac{1}{2} H(2 d+1,2)$;

(iii) the folded cube $\tilde{H}(2 d+1,2)$;

(iv) the dual polar graph on $\left[{ }^{2} A_{2 d-1}(q)\right]$, where $q \geq 2$ is a prime power.

In this note we show that Theorem 1 can be extended to include the diameter four case. The following result is key to doing so.

Theorem 2. Let $\Gamma$ denote a twice Q-polynomial distance-regular graph of diameter four and valency at least three. Then one of the Q-polynomial structures has $a_{1}^{*}=a_{2}^{*}=a_{3}^{*}=0$, that is, this structure is either dual bipartite or almost dual bipartite.

As a consequence of Theorems 1 2 and the classification of distance-regular graphs of diameter at least four that are either dual bipartite or almost dual bipartite [5], we obtain the following result.

Theorem 3. Let $\Gamma$ denote a twice $Q$-polynomial distance-regular graph with diameter $d$ at least 4 and valency at least 3 . Then $\Gamma$ is one of the following:

(i) the cube $H(d, 2)$ with d even;

(ii) the half cube $\frac{1}{2} H(2 d+1,2)$;

(iii) the folded cube $\tilde{H}(2 d+1,2)$;

(iv) the dual polar graph on $\left[{ }^{2} A_{2 d-1}(q)\right]$, where $q \geq 2$ is a prime power;

(v) a Hadamard graph of order $2 \gamma$ with intersection array $\{2 \gamma, 2 \gamma-1, \gamma, 1 ; 1, \gamma, 2 \gamma-1,2 \gamma\}$ with $\gamma=1$ or $\gamma$ a positive even integer.

\footnotetext{
${ }^{*}$ Corresponding author

Email addresses: jianminma@yahoo.com (Jianmin Ma), koolen@ustc.edu.cn (Jack H. Koolen )
} 
Remark 1. It is known that Hadamard graphs of order $2 \gamma$ exist for $\gamma$ any non-negative power of two. A Hadamard graph of order $2 \gamma$ exists if and only if a Hadamard matrix of $2 \gamma$ exists [2, Section 1.8]. A Hadamard matrix of order $n$ with $n$ a positive integer is a square $\{+1,-1\}$ matrix $H$ of order $n$ such that $H H^{T}=n I$. The Hadamard conjecture states that a Hadamard matrix exists if and only if $n=1,2$, or $n$ is a positive integer divisible by 4 .

Distance-regular graphs of diameter 2 are strongly regular graphs, which possess two $P$-polynomial and two $Q$-polynomial structures. Any connected distance regular graph with valency two is an ordinary $n$-gon, which can have more than two $Q$-polynomial structures only if $n \geq 7$. So in the rest of this note, we restrict ourselves to distance-regular graphs with both diameter and valency at least three unless stated otherwise.

\section{Definitions and preliminaries}

In this section, we review some definitions and basic concepts. See the books of Brouwer, Cohen, and Neumaier [2] or Bannai and Ito [1] for more background information.

\subsection{Distance-regular graphs}

Let $\Gamma=(X, R)$ denote a finite, undirected, connected graph, without loops or multiple edges, with vertex set $X$, edge set $R$, path-length distance function $\partial$, and diameter $d=\max \{\partial(x, y) \mid x, y \in X\}$. For all $x \in X$ and for all integers $i$, we set $\Gamma_{i}(x)=\{y \in X \mid \partial(x, y)=i\}$. We abbreviate $\Gamma(x)=\Gamma_{1}(x)$. The valency of a vertex $x \in X$ is the cardinality of $\Gamma(x)$. The graph $\Gamma$ is said to be regular, valency $k$, if each vertex in $X$ has valency $k$. Graph $\Gamma$ is said to be distance-regular whenever for all integers $h, i, j(0 \leq h, i, j \leq d)$, and for all $x, y \in X$ with $\partial(x, y)=h$, the number $p_{i, j}^{h}=\left|\Gamma_{i}(x) \cap \Gamma_{j}(y)\right|$ is independent of $x$ and $y$. When there is no possibility of confusion, we write $p_{i j}^{h}$ instead $p_{i, j}^{h}$. The constants $p_{i j}^{h}$ are known as the intersection numbers of $\Gamma$.

For notational convenience, set $c_{i}=p_{1, i-1}^{i}(1 \leq i \leq d), a_{i}=p_{1 i}^{i}(0 \leq i \leq d), b_{i}=p_{1, i+1}^{i}(0 \leq i \leq d-1), k_{i}=p_{i i}^{0}$ $(0 \leq i \leq d)$, and define $c_{0}=0, b_{d}=0$. We note $a_{0}=0, k_{1}=b_{0}$, and $c_{1}=1$. We write $k=k_{1}$. The sequence

$$
\left\{b_{0}, b_{1}, \ldots, b_{d-1} ; c_{1}, c_{2}, \ldots, c_{d}\right\}
$$

is called the intersection array of $\Gamma$.

From now on, $\Gamma=(X, R)$ will denote a distance-regular graph of diameter $d \geq 3$. Observe that $\Gamma$ is regular with valency $k$, and that

$$
k=c_{i}+a_{i}+b_{i} \quad(0 \leq i \leq d) .
$$

We now recall the Bose-Mesner algebra. For each integer $i(0 \leq i \leq d)$, let $A_{i}$ denote the $i$-th distance matrix with $x, y$ entry

$$
\left(A_{i}\right)_{x y}=\left\{\begin{array}{ll}
1, & \text { if } \partial(x, y)=i, \\
0, & \text { otherwise }
\end{array} \quad(x, y \in X) .\right.
$$

Then

$$
A_{0}=I, \quad A_{0}+A_{1}+\cdots+A_{d}=J, \quad A_{i}^{t}=A_{i}, \quad A_{i} A_{j}=\sum_{k=0}^{d} p_{i j}^{k},
$$

where $J$ is the all-one matrix.

We abbreviate $A=A_{1}$, and refer to this as the adjacency matrix of $\Gamma$. Let $M$ denote the algebra generated by $A$ over the reals $\mathbb{R}$. We refer to $M$ as the Bose-Mesner algebra of $\Gamma$. The matrices $A_{0}, A_{1}, \ldots, A_{d}$ form a basis for $M$. Note that $M$ is closed under the Schur (entry-wise) product $\circ$. So it has a second basis $E_{0}, E_{1}, \ldots, E_{d}$ of primitive idempotents which satisfy

$$
E_{0}=|X|^{-1} J, \quad E_{0}+E_{1}+\ldots E_{d}=I, \quad E_{i}^{t}=E_{i}, \quad E_{i} \circ E_{j}=|X|^{-1} \sum_{k=0}^{d} q_{i j}^{k} E_{k} .
$$

The numbers $q_{i j}^{k}$ are nonnegative reals, and are referred to as Krein parameters. Note the parameters $q_{i j}^{k}$ and $p_{i j}^{k}$ depend on the orderings $\left(E_{i}\right)_{i}$, and $\left(A_{i}\right)_{i}$, respectively.

Let $\theta_{0}, \theta_{1}, \ldots, \theta_{d}$ denote the real numbers satisfying $A=\sum_{i=0}^{d} \theta_{i} E_{i}$. We refer to $\theta_{i}$ as the eigenvalue of $\Gamma$ associated with $E_{i}$, and call $\theta_{0}$ the trivial eigenvalue. If $\theta_{0}>\theta_{1}>\cdots>\theta_{d}$, then we say $E_{0}, E_{1}, \ldots, E_{d}$ is the natural ordering of the primitive idempotents.

A $d$-class association scheme is a pair $\left(X,\left\{R_{0}, \ldots, R_{d}\right\}\right)$ with $R_{i}$ symmetric binary relations on $X$ whose adjacency matrices satisfy (2), where $R_{i}$ has adjacency matrix $A_{i}$ defined by $\left(A_{i}\right)_{x y}=1$ if $(x, y) \in R_{i}$ and 0 otherwise. It also have a set of primitive idempotents that satisfy (3). The property that one of the relations of a $d$-class association scheme forms a distance-regular graph with diameter $d$ is equivalent to the scheme being $P$-polynomial, that is, the relations $R_{0}, \ldots, R_{d}$ can be ordered such that every pair of vertices in $R_{i}$ has distance $i$ in the graph $\left(X, R_{1}\right)$ for every $i$. In turn, this is equivalent to the conditions $p_{1 i}^{i+1}>0$ for $0 \leq i \leq d-1$ and $p_{1 i}^{k}=0$ for $k>i+1$. 


\subsection{Cosines}

We now recall the cosines. Let $\theta$ denote an eigenvalue of $\Gamma$, and let $E$ denote the associated primitive idempotent. Let $\sigma_{0}, \sigma_{1}, \ldots, \sigma_{d}$ denote the real numbers satisfying

$$
E=|X|^{-1} m \sum_{i=0}^{d} \sigma_{i} A_{i},
$$

where $m$ denotes the multiplicity of $\theta$. Taking the trace in (4), we find $\sigma_{0}=1$. We call $\sigma_{i}$ the $i$-th cosine of $\Gamma$ with respect to $\theta$ (or $E$ ), and call $\sigma_{0}, \sigma_{1}, \ldots, \sigma_{d}$ the cosine sequence of $\Gamma$ associated with $\theta$ (or $E$ ).

We will need the following basic results.

Lemma 4. [2, Section 4.1.B] Let $\Gamma$ denote a distance-regular graph with diameter $d \geq 3$. Then for any complex numbers $\theta, \sigma_{0}, \sigma_{1}, \ldots, \sigma_{d}$, the following are equivalent.

(i) $\theta$ is an eigenvalue of $\Gamma$, and $\sigma_{0}, \sigma_{1}, \ldots, \sigma_{d}$ is the associated cosine sequence.

(ii) $\sigma_{0}=1$, and

$$
c_{i} \sigma_{i-1}+a_{i} \sigma_{i}+b_{i} \sigma_{i+1}=\theta \sigma_{i} \quad(0 \leq i \leq d)
$$

where $\sigma_{-1}$ and $\sigma_{d+1}$ are indeterminates.

(iii) $\sigma_{0}=1, k \sigma=\theta$, and

$$
c_{i}\left(\sigma_{i-1}-\sigma_{i}\right)-b_{i}\left(\sigma_{i}-\sigma_{i+1}\right)=k(\sigma-1) \sigma_{i} \quad(1 \leq i \leq d),
$$

where $\sigma_{d+1}$ is an indeterminate.

The second largest and minimal eigenvalue of a distance-regular graph turn out to be of particular interest. In the next several lemmas, we give some basic information on these eigenvalues.

Lemma 5. [8, Lem. 13.2.1] Let $\Gamma$ denote a distance-regular graph with diameter $d \geq 3$, and eigenvalues $\theta_{0}>\theta_{1}>$ $\cdots>\theta_{d}$. Let $\theta$ denote one of $\theta_{1}, \theta_{d}$ and let $\sigma_{0}, \sigma_{1}, \ldots, \sigma_{d}$ denote the cosine sequence for $\theta$.

(i) Suppose $\theta=\theta_{1}$. Then $\sigma_{0}>\sigma_{1}>\cdots>\sigma_{d}$.

(ii) Suppose $\theta=\theta_{d}$. Then for each $i(0 \leq i \leq d),(-1)^{i} \sigma_{i}>0$.

Lemma 6. Let $\Gamma$ be a distance-regular graph with diameter $d \geq 3$ and eigenvalues $\theta_{0}>\theta_{1}>\cdots>\theta_{d}$. Then (i)-(iii) below hold.

(i) $0<\theta_{1}<k$.

(ii) $a_{1}-k \leq \theta_{d}<-1$.

(iii) If $\Gamma$ is not bipartite, then $a_{1}-k<\theta_{d}$.

\subsection{Q-polynomial property}

Let $\theta_{0}, \theta_{1}, \ldots, \theta_{d}$ (or $\left.E_{0}, E_{1}, \ldots, E_{d}\right)$ be a fixed ordering of the eigenvalues (or primitive idempotents) of $\Gamma$. We call this ordering is a $Q$-polynomial structure if there is a sequence $\sigma=\left(\sigma_{0}, \sigma_{1}, \ldots, \sigma_{d}\right)$ and polynomial $q_{j}$ of degree $j, j=0,1, \ldots, d$, such that

$$
E_{j}=\sum_{i=0}^{d} q_{j}\left(\sigma_{i}\right) A_{i} ;
$$

in this case, $\sigma$ is called a $Q$-sequence of $\Gamma$ and $E_{1}$ is called the primary idempotent for this $Q$-sequence. The graph $\Gamma$ is called $Q$-polynomial if $\Gamma$ has a $Q$-polynomial structure.

Let $E_{0}, E_{1}, \ldots, E_{d}$ be a $Q$-polynomial structure for $\Gamma$. We usually write $a_{i}^{*}=q_{1 i}^{i}, b_{i}^{*}=q_{1, i+1}^{i}, c_{i}^{*}=q_{1, i-1}^{i}$ and $k_{i}^{*}=q_{i i}^{0}$ for $i=0,1, \ldots, d$.

We will need the following theorem of H. Suzuki.

Theorem 7. [18] Suppose that $\mathcal{X}$ is a symmetric association scheme with a $Q$-polynomial structure $E_{0}, E_{1}, \ldots, E_{d}$. If $X$ is not a polygon and has another $Q$-polynomial structure, then the new structure is one of the following:

(I) $E_{0}, E_{2}, E_{4}, E_{6}, \ldots, E_{5}, E_{3}, E_{1}$

(II) $E_{0}, E_{d}, E_{1}, E_{d-1}, E_{2}, E_{d-2}, E_{3}, E_{d-3}, \ldots$;

(III) $E_{0}, E_{d}, E_{2}, E_{d-2}, E_{4}, E_{d-4}, \ldots, E_{d-5}, E_{5}, E_{d-3}, E_{3}, E_{d-1}, E_{1}$;

(IV) $E_{0}, E_{d-1}, E_{2}, E_{d-3}, E_{4}, E_{d-5}, \ldots, E_{5}, E_{d-4}, E_{3}, E_{d-2}, E_{1}, E_{d}$;

(V) $d=5$ and $E_{0}, E_{5}, E_{3}, E_{2}, E_{4}, E_{1}$.

Hence, $X$ admits at most two $Q$-polynomial structures.

Case (V) was recently eliminated in [15]. 


\subsection{Almost dual primitivity}

The graph $\Gamma$ is called imprimitive when some $i, 1 \leq i \leq d$, the distance- $i$ graph $\Gamma_{i}=\left(X, A_{i}\right)$ is disconnected. If $\Gamma$ is imprimitive, then by 2, Theorem 4.2.1], $\Gamma$ is bipartite (here $\Gamma_{2}$ is disconnected) or antipodal (here $\Gamma_{d}$ is a union of cliques).

A $Q$-polynomial structure $\left(E_{i}\right)_{i=0}^{d}$ is called dual bipartite if $a_{0}^{*}=a_{1}^{*}=\cdots=a_{d}^{*}=0$. When there is no possibility of confusion, we also say that graph $\Gamma$ is dual bipartite. Similar comment applies the other concepts to follow immediately. If $c_{i}^{*}=b_{d-i}^{*}$ for $i=0,1, \ldots, d$ and $i \neq\lfloor d / 2\rfloor$, then $\Gamma$ is called dual antipodal. An imprimitive $Q$-polynomial distance-regular graph is either dual bipartite or dual antipodal (or both).

Now we define the terms of almost dual bipartite/antipodal, introduced by Dickie [5]. A $Q$-polynomial structure $\left(E_{i}\right)_{i=0}^{d}$ is called almost dual bipartite if $a_{0}^{*}=a_{1}^{*}=\cdots=a_{d-1}^{*}=0 \neq a_{d}^{*}$; it is called almost dual antipodal if $q_{1 d}^{d} \neq 0=q_{2 d}^{d}=\cdots=q_{d d}^{d}$. If $\Gamma$ is almost dual bipartite or antipodal, then it is called almost dual imprimitive.

For a classification of almost dual imprimitive distance-regular graphs, see [5, Theorem 3.1.4] for the almost dual bipartite case with $d \geq 4$, and [5, Theorem 2.1.2], ,7] for the dual bipartite case with $d \geq 3$.

\subsection{The tight property}

Now we recall the tight property [9]. A distance-regular graph $\Gamma$ is called tight if it is not bipartite and the following equality holds

$$
\left(\theta_{1}+\frac{k}{a_{1}+1}\right)\left(\theta_{d}+\frac{k}{a_{1}+1}\right)=-\frac{k a_{1} b_{1}}{\left(a_{1}^{2}+1\right)^{2}},
$$

where $\theta_{1}$ and $\theta_{d}$ are the second largest and the smallest eigenvalues of $\Gamma$, respectively.

\section{Proof of Theorem 3}

We prove our main theorems in this section. In the rest of this paper, we will fix $\Omega$ to be a general distance-regular graph and $\Gamma$ to be a twice $Q$-polynomial distance-regular graph of diameter 4 . Let $E_{0}, \ldots, E_{4}$ and $\tilde{E}_{0}, \ldots, \widetilde{E}_{4}$ be $Q$-polynomial structures for $\Gamma$. The parameters for $\left(\tilde{E}_{i}\right)_{i}$ will be attached with a tilde.

We first prove Theorem 2, which is key to the proof of Theorem 3 .

\subsection{Proof of Theorem 2}

We first quote some results from Dickie's thesis [5].

Theorem 8. [5, Theorem 7.1.1], [6] Let $\Omega=(X, R)$ denote a distance regular graph with diameter $d \geq 3$. Suppose that $\Omega$ admits more than one $Q$-polynomial structure. Then $\Omega$ is thin and dual thin.

The follow result follows from Theorem 8 and Theorems 4.1.1 and 5.1.1. in [5].

Theorem 9. [5, Theorem 5.1.2] Let $\Omega$ be as in Theorem 8 with a Q-polynomial structure $E_{0}, E_{1}, \ldots, E_{d}$, Krein parameters $q_{1 i}^{i}$ and intersection numbers $p_{1 i}^{i}$. Then we have the following implications:

$$
\begin{array}{lll}
q_{11}^{1}=0 & \Rightarrow & q_{1 i}^{i}=0 \\
q_{11}^{1} \neq 0 & \Rightarrow & q_{1 i}^{i} \neq 0 \\
p_{11}^{1}=0 & \Rightarrow & p_{1 i}^{i}=0
\end{array}
$$

for all $i=1,2, \ldots, d-1$.

The dual of (8), i.e., $p_{11}^{1} \neq 0 \Rightarrow p_{1 i}^{i} \neq 0,1 \leq i \leq d-1$, holds for any distance-regular graph [2, p.178]. By [5, Corollary 6.2.4], the graph $\Omega$ in Theorem 8 is locally strongly regular: if for $x \in X, \Omega(x)$ is the set of vertices adjacent to $x$, the induced graph on $\Omega(x)$ is strongly regular.

If $\Gamma$ has $q_{11}^{1}=0$ (or $\tilde{q}_{11}^{1}=0$ ), then, by (77), it is dual bipartite in case $q_{1 d}^{d}=0$ (or $\tilde{q}_{11}^{1}=0$ ) and almost dual bipartite otherwise.

The following result applies when $q_{11}^{1} \neq 0$ and $\tilde{q}_{11}^{1} \neq 0$.

Lemma 10. [5, Lemma 8.2.1] Let $\Omega$ be distance-regular graph with diameter $d \geq 4$. Suppose $E_{0}, E_{1}, \ldots, E_{d}$ and $\tilde{E}_{0}, \tilde{E}_{1}, \ldots, \tilde{E}_{d}$ are $Q$-polynomial structures for $\Omega$, with Krein parameters $q_{i j}^{k}$ and $\tilde{q}_{i j}^{k}$, respectively. If $q_{11}^{1} \neq 0$ and $\tilde{q}_{11}^{1} \neq 0$, then $E_{1}=\tilde{E}_{d}, E_{d}=\tilde{E}_{1}$ and $d=4$.

By Lemma 10, $\tilde{E}_{1}=E_{4}$ and $\tilde{E}_{4}=E_{1}$. By Theorem 7 the $Q$-polynomial structures for $\Gamma$ have type III. The following hold by the $Q$-polynomial property (see also [18, Theorem 2]):

$$
q_{14}^{4}=0=q_{34}^{4}, \quad q_{24}^{4} \neq 0 \neq q_{23}^{4} .
$$

Pascasio [16] showed the following results. 
Theorem 11. [16, Theorem 1.3, Lemma 3.2] Let $\Omega$ be a distance regular graph with diameter $d \geq 3$ and eigenvalues $\theta_{0}>\theta_{1}>\cdots>\theta_{d}$. Let $E$ and $F$ be two primitive idempotents other than $E_{0}$.

(i) Suppose $\Omega$ is tight. Then $E \circ F$ is a scalar multiple of a primitive idempotent $H$ of $\Omega$ if and only if $E, F$ are a permutation of $E_{1}, E_{d}$. Moreover, the scalar is $\frac{m_{E} m_{F}}{|X| m_{H}}$.

(ii) Suppose $\Omega$ is bipartite. Then $E \circ F$ is a scalar multiple of a primitive of $\Omega$ if and only if at least one of $E, F$ is equal to $E_{d}$.

(iii) Suppose $\Omega$ is neither bipartite nor tight. Then $E \circ F$ is never a scalar multiple of a primitive of $\Omega$.

Theorem 12. [1], Theorem 1.3] Let $\Omega$ be a Q-polynomial distance-regular graph with diameter $d \geq 3$, intersection numbers $a_{i}$ and Krein parameters $a_{i}^{*}, 0 \leq i \leq d$. The following are equivalent.

(i) $\Gamma$ is tight.

(ii) $\Gamma$ is not bipartite and $a_{d}=0$.

(iii) $\Gamma$ is not bipartite and $a_{d}^{*}=0$.

Since $q_{14}^{4}=0, E_{1} \circ E_{4}=|V \Gamma|^{-1} b_{3}^{*} E_{3}$, where $V \Gamma$ is the vertex set of $\Gamma$. Theorem 11 says that $\theta_{4}$ is the eigenvalue associated with $E_{4}$ or $\tilde{E}_{4}$. Without loss of generality, we assume that it is $E_{4}$. If $\Gamma$ is tight, then $E_{1}$ is associated with $\theta_{1}$. By [17, Theorem 1.5], $E_{0}, E_{1}, E_{2}, E_{3}, E_{4}$ is the natural ordering of the primitive idempotents. Now we denote the eigenvalues of $\Gamma$ by $\theta_{0}>\theta_{1}>\theta_{2}>\theta_{3}>\theta_{4}$.

Now we prove Theorem 2 by showing that one of $a_{1}^{*}, \tilde{a}_{1}^{*}$ for $\Gamma$ vanishes. We distinguishing whether $\Gamma$ is bipartite or not.

\subsection{1. $\Gamma$ is bipartite}

Assume that $\Gamma$ is bipartite. So we have $\theta_{4}=-k$ and $m_{4}=1$. Imprimitive $Q$-polynomial association schemes have the following characterization.

Theorem 13. ([19, Theorem 3], [3, 20]) Let $E_{0}, E_{1}, \ldots, E_{d}$ be a Q-polynomial structure for association scheme $X$. Suppose that $\mathcal{X}$ is imprimitive. More precisely, let $T$ be a proper subset of $\{0,1, \ldots, d\}$ with $T \neq\{0\}$ such that the linear span of $\left\{E_{i} \mid i \in T\right\}$ is closed under the Schur product. In addition, assume $m_{1}>2$. Then one of following holds:

(i) $T=\{0,2,4, \ldots\}$ and $a_{i}^{*}=0$.

(ii) $T=\{0, d\}$ and $b_{i}^{*}=c_{d-i}^{*}$ for all $i=0,1, \ldots, d$ with the possible exception $i=\lfloor d / 2\rfloor$.

An association scheme $X$ in case (ii) and (iii) is also called dual bipartite and dual antipodal respectively.

Now back to $\Gamma$. Let $m_{1}=m_{E_{1}}$. Suppose $m_{1}>2$. Then Theorem 13 applies. If $q_{11}^{1}>0, \Gamma$ can not be dual bipartite and hence $\Gamma$ is dual antipodal, i.e., case (ii). So $T=\{0,4\}$. However, $E_{4}$ is the primary idempotent for the second $Q$-polynomial structure, which is impossible.

Suppose $m_{1} \leq 2$. Since $m_{E_{1}}<k=3$, we have $\theta_{E_{1}}=\theta_{1}$ by [2, Theorem 4.4.4]. If $m_{1}=2$, then by [14, Theorem 13 (i)] $k=2$, this contradicts $k>2$. By [14, Lemma 7], it is impossible for $m_{1}=1$; otherwise $m_{1}+m_{4}=2<k$.

\subsection{2. $\Gamma$ is not bipartite}

Assume that $\Gamma$ is not bipartite. Since $a_{4}^{*}=0, \Gamma$ is tight and $a_{4}=0$ by Theorem [12 In the literature [2, p.247], there is an infinite series of feasible formally self-dual intersection arrays

$$
\left\{\mu(2 \mu+1),(\mu-1)(2 \mu+1), \mu^{2}, \mu ; 1, \mu, \mu(\mu-1), \mu(2 \mu+1)\right\}
$$

This series was ruled out in [11]. Had a graph with this array existed, it would be tight with a pair of non-integral eigenvalues, and would have possessed two $P$-polynomial and two $Q$-polynomial structures. We will show below that there are no distance-regular graphs with intersection array (10) in an alternative way.

Since $\Gamma$ is not bipartite, it follows from (8) that $a_{1} a_{2} a_{3} \neq 0, a_{4}=0$. We can deduce from Theorem 11 (i) and Lemma 4 (iii) that

$$
\theta_{1} \theta_{4}=\theta_{0} \theta_{3} .
$$

Theorem 14. [G, Theorem 8.1.2, Corollary 8.1.4] Let $\Omega$ be a Q-polynomial distance-regular graph. Then every $Q$-sequence $\left(\sigma_{0}, \sigma_{1}, \ldots, \sigma_{d}\right)$ of $\Omega$ satisfies the recurrence

$$
\sigma_{i+1}+\sigma_{i-1}=p \sigma_{i}+r \quad(i=1, \ldots, d-1),
$$

for suitable numbers $p$ and $r$.

If $k=\theta_{0}, \theta_{1}, \ldots, \theta_{d}$ the $Q$-polynomial structure corresponding to the above $Q$-sequence, then there are constants $r^{*}, s^{*}$ such that

$$
\left.\begin{array}{l}
\theta_{\ell+1}+\theta_{\ell-1}=p \theta_{\ell}+r^{*} \\
\theta_{\ell+1} \theta_{\ell-1}=\theta_{\ell}^{2}-r^{*} \theta_{\ell}-s^{*}
\end{array}\right\} \quad(\ell=1, \ldots, d-1) .
$$


Now applying Theorem 14 to the two $Q$-polynomial structures of $\Gamma$, we obtain:

$$
\begin{aligned}
& \theta_{2}-p \theta_{1}+\theta_{0}=\theta_{3}-p \theta_{2}+\theta_{1}=\theta_{4}-p \theta_{3}+\theta_{2} \\
& \theta_{2}-\tilde{p} \theta_{4}+\theta_{0}=\theta_{3}-\tilde{p} \theta_{2}+\theta_{4}=\theta_{1}-\tilde{p} \theta_{3}+\theta_{2}
\end{aligned}
$$

We obtain from (14) and (15)

$$
p=\frac{\theta_{0}-\theta_{4}}{\theta_{1}-\theta_{3}}, \quad \tilde{p}=\frac{\theta_{0}-\theta_{1}}{\theta_{4}-\theta_{3}}, \quad p-\tilde{p}=\frac{2\left(\theta_{1}-\theta_{4}\right)}{\theta_{2}-\theta_{3}} .
$$

From these equation and $\theta_{1} \theta_{4}=k \theta_{3}$ we find

$$
p=\theta_{0} / \theta_{1}, \quad \tilde{p}=\theta_{0} / \theta_{4}, \quad \theta_{2}=-\theta_{3} .
$$

Now substituting these into (14) leads to

$$
\theta_{1}+\theta_{4}=2 \theta_{2}
$$

If we substitute (16) into [2, Corollary 8.1.4 (24)], we find

$$
r^{*}=\theta_{2}, \quad s^{*}=\theta_{1} \theta_{3}, \quad \tilde{r}^{*}=\theta_{2}, \quad \tilde{s}^{*}=\theta_{4} \theta_{2} .
$$

Since $\Gamma$ is tight, we have

$$
\left(\theta_{1}+\frac{k}{a_{1}+1}\right)\left(\theta_{4}+\frac{k}{a_{1}+1}\right)=-\frac{k a_{1} b_{1}}{\left(a_{1}+1\right)^{2}} .
$$

We find from this and Eq. (17) and (11) that

$$
\theta_{2}\left(a_{1}-1\right)=b_{1}+1
$$

Now we collect some equations above that are key to the proof to follow:

$$
\begin{aligned}
\theta_{1} \theta_{4} & =\theta_{0} \theta_{3} \\
\theta_{1}+\theta_{4} & =2 \theta_{2} \\
\theta_{2}\left(a_{1}-1\right) & =b_{1}+1 .
\end{aligned}
$$

Since $\Gamma$ is tight, a local graph $\Gamma(x)$ is strongly regular with $k$ vertices and valency $a_{1}$ and non-trivial eigenvalues

$$
\xi=-1-\frac{b_{1}}{\left(\theta_{4}+1\right)}, \quad \tau=-1-\frac{b_{1}}{\left(\theta_{1}+1\right)} .
$$

where $\xi \geq 0$ and $\tau<-1$.

The local graph $\Gamma(x)$ can not be a conference graph. Otherwise, we have $a_{1}=(k-1) / 2$ and such a graph has diameter 3 by [13]. (The intersection array (10) has the second largest and minimal eigenvalues non-integral, and thus any graph with this array has a conference graph as its local graph and therefore can not exist.) Therefore, $\sigma$ and $\tau$ are both integers and thus $\theta_{4}, \theta_{1}$ are both rational numbers. Since they are algebraic integers, $\theta_{4}, \theta_{1}$ are integers.

We find from (201)-(22) that

$$
-\left(\theta_{1}+1\right)\left(\theta_{4}+1\right)=-\theta_{1} \theta_{4}-\theta_{1}-\theta_{2}-1=(k-2) \theta_{2}-1=\left(a_{1}-1\right) \theta_{2}+b_{1} \theta_{2}-1=b_{1}+1+b_{1} \theta_{2}-1=b_{1}\left(\theta_{2}+1\right) .
$$

From this we can derive

$$
\frac{-b_{1}^{2}}{\left(\theta_{1}+1\right)\left(\theta_{4}+1\right)}=\frac{b_{1}}{\left(\theta_{2}+1\right)} .
$$

Since the left hand side is an integer, $\theta_{2}+1$ divides $b_{1}$ and hence $a_{1}$ by (22).

Let $\left(\sigma_{i}\right)_{i}$ be the cosine sequence of $\theta_{1}$. Then $\sigma_{i}>\sigma_{i+1}(0 \leq i \leq 3)$ and $\sigma_{3}=\sigma_{1} \sigma_{4}$. As the sequence $\sigma_{i}$ has one sign change, $\sigma_{4}<0$ and $\sigma_{1}>0$. Hence $\sigma_{3}<0$. Let $\left(\tilde{\sigma}_{i}\right)_{i}$ be cosine sequence of $\theta_{4}$. Then $(-1)^{i} \tilde{\sigma}_{i}>0$. Let $\left(u_{i}\right)_{i}$ be the cosine sequence of $\theta_{3}$. Then $u_{i}=\sigma_{i} \tilde{\sigma}_{i}$.

Let $a_{1}=\alpha\left(\theta_{2}+1\right), b_{1}=\beta\left(\theta_{2}+1\right)$. By (20) and (21) we find $\theta_{1}, \theta_{4}=\theta_{2} \pm \sqrt{\theta_{2}\left(k+\theta_{2}\right)}$. Now $\theta_{2}\left(k+\theta_{2}\right)=\alpha \theta_{2}\left(\theta_{2}+1\right)^{2}$ and hence $k=\alpha\left(\theta_{2}+1\right)^{2}-\theta_{2}$.

By [4, Proposition 1 (iii), Proposition 2 (iii)], $\sigma_{2} \geq 0$ and thus $u_{2} \geq 0$. We find from this and $\theta_{2}=-\theta_{3}$ that $k-\theta_{2} a_{1} \leq \theta_{2}^{2}$. As $k=\left(a_{1}-1\right)\left(\theta_{2}+1\right)+1$, we obtain $a_{1} \leq \theta_{2}\left(\theta_{2}+1\right)$ and thus $\alpha \leq \theta_{2}$.

Recall $\left(\alpha\left(\theta_{2}+1\right)-1\right) \theta_{2}-1=\left(a_{1}-1\right) \theta_{2}-1=b_{1}=\beta\left(\theta_{2}+1\right)$. We obtain: $\beta=\alpha \theta_{2}-1$ and $b_{1}=\left(\alpha \theta_{2}-1\right)\left(\theta_{2}+1\right)$, $\xi=-1-b_{1} /\left(\theta_{4}+1\right)=\sqrt{\alpha \theta_{2}}$ and $\tau=-\xi$. This means that $\xi$ divides $a_{1}\left(=k-b_{1}-1\right)$ and hence, as $a_{1}=\xi^{2}+\alpha$, 
$\xi$ divides $\alpha$. This implies that $\theta_{2}$ divides $\alpha$ and hence $\theta_{2} \leq \alpha$. We conclude $\alpha=\theta_{2}=\xi$. Now, the local graph $\Gamma(x)$ has the following parameters:

$$
k=\theta_{2}^{2}\left(\theta_{2}+2\right), \quad a_{1}=\theta_{2}\left(\theta_{2}+1\right), \quad \theta_{1}=\theta_{2}\left(\theta_{2}+2\right), \quad \theta_{4}=-\theta_{2}^{2} .
$$

We see that

$$
\theta_{1}=\frac{a_{1}+\sqrt{a_{1}^{2}+4 k}}{2}
$$

Now $\Gamma$ is antipodal by the following result.

Lemma 15. [12, Proposition 3.5] Let $\Omega$ be a distance-regular graph with $d$ at least three and distinct eigenvalues $k=\theta_{0}>\theta_{1}>\cdots>\theta_{d}$. Then $\theta_{1}=\left(a_{1}+\sqrt{a_{1}^{2}+4 k}\right) / 2$ if and only if one of the following holds:

(i) $d=3$ and $\Omega$ is a Shilla distance-regular graph;

(ii) $d=4$ and $\Omega$ is an antipodal distance-regular graph.

Since $\Gamma$ is antipodal, it is dual bipartite. So $a_{1}^{*}=0$, or $\tilde{a}_{1}^{*}=0$. This completes the proof of Theorem 2 .

\subsection{Proof of Theorem 3}

By Lemma 9 and the succeeding remark, the parameters $a_{i}^{*}$ of $\Gamma$ can be divided into the following four cases:

(i) If $a_{1}^{*}=a_{4}^{*}=0$, then $\Gamma$ is $H(4,2)$ or a Hadamard graph by [7].

(ii) If $a_{1}^{*}=0 \neq a_{4}^{*}$, then $\Gamma$ is $\frac{1}{2} H(9,2)$, or $\tilde{H}(9,2)$ by [5, Theorem 3.1.4].

(iii) Suppose $a_{1}^{*} \neq 0 \neq a_{4}^{*}$. By [18, Theorem 2], the $Q$-polynomial structures $\left(E_{i}\right)_{i}$ is almost dual antipodal and thus by [5, Lemma 3.1.3], the other $Q$-polynomial structure $\left(\tilde{E}_{i}\right)_{i}$ is almost dual bipartite. This case is implied by the previous case by treating $\left(\tilde{E}_{i}\right)_{i}$, which has $\tilde{a}_{1}^{*}=0 \neq \tilde{a}_{4}^{*}$.

(iv) If $a_{1}^{*} \neq 0=a_{4}^{*}$, then $\tilde{a}_{1}^{*}=0$ by Theorem 2 and Cases (i),(ii) apply.

So the proof of Theorem 3 is completed.

Remark 2. The following graphs are twice $Q$-polynomial distance-regular graphs of diameter 3: each graph in Theorem 1 (ii)-(iv) with $d=3$, and a distance-regular graph intersection array $\left\{k, k-a_{1}-1,1 ; 1, k-a_{1}-1, k\right\}$. If $a_{1}=0$, this array is uniquely realized by the complement of $K_{k+1} \times K_{2}$; any distance-regular graph with this intersection array is called a Taylor graph if $a_{1}>0$; see [2, p.13].

\section{Acknowledgments}

J. Koolen thanks the 100 Talents Program of the Chinese Academy of Sciences for support. J. Ma acknowledges support from the Natural Science Foundation of Hebei province (A2012205079) and Science Foundation of Hebei Normal University (L2011B02).

\section{References}

[1] E. Bannai, T. Ito, Algebraic Combinatorics I: Association Schemes, The Benjamin/Cummings Publishing Company, Inc., Menlo Park, CA, 1984.

[2] A. E. Brouwer, A. M. Cohen, A. Neumaier, Distance-regular graphs, Springer, New York, 1989.

[3] D. R. Cerzo, H. Suzuki, Non-existence of imprimitive $Q$-polynomial schemes of exceptional type with $d=4$, European J. Combin. 30 (2009) 674-681.

[4] S. M. Cioabă, J. H. Koolen, On the connectedness of the complement of a ball in distance-regular graphs, J. Algebraic Combin. 38 (2013) 191-195.

[5] G. A. Dickie, $Q$-polynomial structures for association schemes and distance regular graphs, PhD thesis, University of Wisconsin-Madison, 1995.

[6] G. A. Dickie, Twice Q-polynomial distance-regular graphs are thin, European J. Combin. 16 (1995) 555-560.

[7] G. A. Dickie, P. Terwilliger, dual bipartite Q-polynomial distance-regular graphs, European J. Combin. 17 (1996) 613-623.

[8] C. D. Godsil, Algebraic Combinatorics, Chapman and Hall, New York, 1993.

[9] A. Jurišić, J. Koolen, P. Terwilliger, Tight distance-regular graphs, J. Algebraic Combin. 12 (2000) 163-197. 
[10] A. Jurišić, P. Terwilliger, A. Žitnik, Distance-regular graphs with light tails, European J. Combin. 31 (2010) $1539-1552$.

[11] J. H. Koolen, C. D. Godsil, On the multiplicity of eigenvalues of distance-regular graphs, Linear Algebra Appl. 226-228 (1995) 273-275.

[12] J. H. Koolen, J. Park, H. Yu, An inequality involving the second largest and smallest eigenvalue of a distanceregular graph, Linear Algebra Appl. 434 (2011) 2404-2412.

[13] J. H. Koolen, J. Park, Distance-regular graphs with $a_{1}$ or $c_{2}$ at least half the valency, J. Combin. Theory Ser. A 119 (2012) 546-55.

[14] J. H. Koolen, J. Kim, J. Park, Distance-regular graphs with a relatively small eigenvalue multiplicity, Elec. J. Combin. 20:1 (2013), paper \#1.

[15] J. Ma, K. Wang, The nonexistence of exceptional 5-class association schemes with two $Q$-polynomial structures, Linear Algebra Appl. 440 (2014) 278-285.

[16] A. Pascasio, Tight graphs and their primitive idempotents, J. Algebraic Combin. 10 (1999) 47-59.

[17] A. Pascasio, Tight distance-regular graphs and the Q-polynomial property, Graph. Combin. 17 (2001) 149-169.

[18] H. Suzuki, Association schemes with multiple $Q$-polynomial structures, J. Algebraic Combin. 7 (1998) 181-196.

[19] H. Suzuki, Imprimitive Q-polynomial association schemes, J. Algebraic Combin. 7(1998) 165-180.

[20] H. Tanaka, R. Tanaka, Nonexistence of exceptional imprimitive $Q$-polynomial association schemes with six classes, European J. Combin. 32 (2011) 155-161. 